\title{
Apoio a Reutilização de Processos de Software através de Templates e Versões
}

\author{
Anderson Costa, Ernani Sales, Carla A. Lima Reis, Rodrigo Quites Reis
}

\author{
Programa de Pós-Graduação em Ciência da Computação \\ Laboratório de Engenharia de Software - Universidade Federal do Pará (UFPA)

$$
\begin{gathered}
\text { Belém-PA-Brasil } \\
\text { \{anderson, ernani, clima, quites\}@webapsee.com }
\end{gathered}
$$

\begin{abstract}
This paper presents the WebAPSEE-Reuse tool - an extension to an existing software process management environment - in order to provide automated support for software reuse. This extension is based on the template construct as a reusable building block that evolves in a controllable manner. The text also provides an evaluation of the tool to specify a generic template based on the Rational Unified Process, which is used to enable further discussion about the practice on the use of the proposed customization facilities.
\end{abstract}

Resumo. Este artigo apresenta a ferramenta WebAPSEE-Reuse - uma extensão do ambiente de gestão de processos de software - a qual é voltada para prover apoio automatizado à reutilização de processos de software. A extensão é baseada no conceito de templates (gabaritos) de processos, os quais têm sua evolução gerenciada em versões. O artigo apresenta ainda uma avaliação da ferramenta baseada no seu uso para especificação do modelo Rational Unified Process, além de discutir seu uso prático na customização manual de modelos.

\section{Introdução}

Segundo Pressman (2005), nos dias atuais, o software constitui a base de sustentação e/ou diferencial de inúmeras organizações nos diversos ramos de atuação ao redor do mundo. Nesse contexto, tornam-se importantes os problemas enfrentados pela indústria de software acerca da baixa qualidade e produtividade dos softwares desenvolvidos para um mercado consumidor cada vez mais exigente.

Segundo a experiência da indústria de software, tais problemas são resultado da falta de um processo que gerencie e controle a qualidade do software desenvolvido [Rocha 2000]. Além disso, diversos pesquisadores da literatura especializada da área como Fuggetta (2000) - afirmam que existe uma relação diretamente proporcional entre a qualidade de um software produzido e o processo de desenvolvimento seguido para a sua construção.

A reutilização de processos de software pode trazer economias, tanto no custo quanto no prazo de entrega na produção de produtos de software em série, quando comparado com produtos independentemente produzidos. Do mesmo modo, a busca pela melhoria de processos, representada pelo surgimento dos modelos de qualidade do processo - tais como o CMMI [SEI 2007], o ISO-SPICE [Dorling et al 2007] e, no Brasil, o MPS.BR 
[Softex 2007]) - enfatiza a definição de processos padrão da organização, permitindo sua adaptação e melhoria contínua para aumentar a qualidade do processo e do produto de software desenvolvido.

Apesar dos fortes argumentos favoráveis à reutilização de processos de software, apenas uma pequena parte do conhecimento produzido é devidamente registrada para ser posteriormente recuperada em novos projetos. Além das especificações de requisitos, projetos, programas, documentos, casos de teste e outros tipos de artefatos comumente produzidos, o conhecimento adquirido sobre o domínio da aplicação a partir das decisões gerenciais - tais como a política de alocação de recursos e o escalonamento das atividades - devem ser registrados para definir uma base de conhecimento que seja reutilizável em projetos futuros [Reis 2002]. Dessa forma, devese criar um cenário em que todas essas informações sejam armazenadas, isto é, dinamicamente adicionadas, excluídas, substituídas e versionadas em um repositório de processos de software, para serem recuperadas e reutilizadas pela organização de acordo com novos requisitos e aperfeiçoamentos gerados a partir de novas idéias.

A ferramenta WebAPSEE-Reuse aqui descrita fornece um conjunto de mecanismos para apoiar a definição de processos reutilizáveis em um repositório, tendo sido construída para atender os desafios enumerados acima. Tais mecanismos foram construídos sobre o ambiente de gestão de processos de software WebAPSEE, que é um Software Livre em desenvolvimento pelo Laboratório de Engenharia de Software da Universidade Federal do Pará [Costa 2006].

O texto é organizado como segue. A seção 2 apresenta a ferramenta WebAPSEE-Reuse. A seção 3 descreve um estudo de caso realizado com um processo consolidado da indústria de software, o Rational Unified Process (RUP) [Kruchten 2000], para avaliar os mecanismos propostos pela ferramenta. Na seção 4, são discutidos os trabalhos correlatos ao apresentado nesse artigo. Finalmente, a seção 5 apresenta as considerações finais do texto.

\section{O Modelo Proposto - WebAPSEE-Reuse}

Primeiramente, essa seção discute a importância do assunto reutilização de processo de software, atrelando sua descrição às demandas dos modelos de maturidade e às características do suporte tecnológico existente. Em seguida, é apresentado o histórico da ferramenta WebAPSEE e as motivações para a sua construção. Posteriormente, são destacadas as conformidades da ferramenta com um dos importantes modelos de qualidade de processos de software, o MPS.BR [Softex 2007] em termos de áreas-chave de processo. E, por fim, apresenta as funcionalidades providas pela ferramenta do ponto de vista do usuário final.

\subsection{Motivação para o Reuso de Processos de Software}

Segundo Krueger (1992), reutilização de software é o processo de criação de software a partir de produtos existentes ao invés de construí-los desde o início. Além disso, Mohagheghi (2004) afirma que reutilizar software reduz o tempo de produção e aumenta a qualidade e confiabilidade do software produzido. Dessa forma, a produtividade aumenta à medida que soluções de problemas passados possam ser aproveitadas para resolver novos problemas. A partir dessas idéias, a Reutilização de Processos de Software vêm se destacando como uma das principais práticas para prover 
a melhoria contínua de processos por aproveitar as informações (tecnológicas e gerencias) produzidas durante desenvolvimentos de software passados, com o objetivo de reduzir o esforço necessário para o desenvolvimento de um novo projeto. Dessa forma, as organizações de desenvolvimento de software podem obter expressivas economias, além de permitir um efetivo aumento na qualidade do software produzido.

Os modelos de qualidade de software - como o CMMI, ISO-SPICE e o MPS.BR - têm como base: 1) a definição de um processo de software padrão, isto é, um processo comum para todos os projetos da organização; 2) know-how para adaptá-lo de acordo com características específicas desses projetos; 3) além da capacidade de prover a melhoria contínua do processo padrão para atender as mudanças do contexto empresarial [Fiorini 2001]. Portanto, todo esforço realizado para auxiliar na reutilização de processos de software contribui para um aumento na maturidade organizacional.

Mais especificamente, pode-se citar que o MPS.BR lida explicitamente com o reuso de processos a partir do nível E (Parcialmente Definido). Neste nível são estabelecidos dois processos fortemente vinculados com o tema, os quais são: a "Adaptação do Processo para Gerência do Projeto" (APG) e "Definição do Processo Organizacional" (DFP).

\subsection{Auxílio por Computador ao Reuso de Processos de Software}

A figura 1 representa o ciclo de vida de processos de software simplificado e orientado de acordo com a ótica da reutilização dos modelos proposta por Jørgensen (2001). Trata-se de uma visão sob o aspecto tecnológico daquilo que é preconizado pelos modelos de maturidade citados na seção anterior. Embora haja uma grande diversidade de ferramentas e soluções tecnológicas que possam ser usadas no contexto do reuso de processos de software, a visão de Jørgensen é útil por estabelecer uma taxonomia comum para as etapas que podem ser automatizadas.

Na figura 1, as setas são etapas, enquanto que os vértices, rotulados em itálico, são estados. Sendo assim, a etapa Modelagem de Processos Visando a Reutilização tem como objetivo definir, através de uma linguagem, os modelos de processos bem como componentes genéricos e abstratos que possam ser reutilizados em diferentes contextos. A etapa Recuperação e Adaptação de Processos prevê critérios, estratégias e mecanismos para auxiliar um projetista na seleção, adaptação e instanciação de processos genéricos que se tornem soluções para os problemas vigentes. A etapa Execução de Processos descreve todas as ocorrências de um modelo de processo desde o início de sua execução, incluindo as modificações referentes à evolução do processo. Por fim, a Generalização e Avaliação de Processos Encerrados extrai informações a partir da experiência adquirida com processos bem sucedidos para alimentar o repositório de modelos de processos reutilizáveis.

O trabalho aqui apresentado está centrado na descrição dos mecanismos propostos para atender a produção de processos genéricos. Embora as funcionalidades oferecidas contribuam - com diferentes níveis de automação - para todas as etapas do ciclo de reuso de processos mostrado acima, este texto prossegue com a descrição mais voltada à descrição à Modelagem de Processos Visando a Reutilização. 


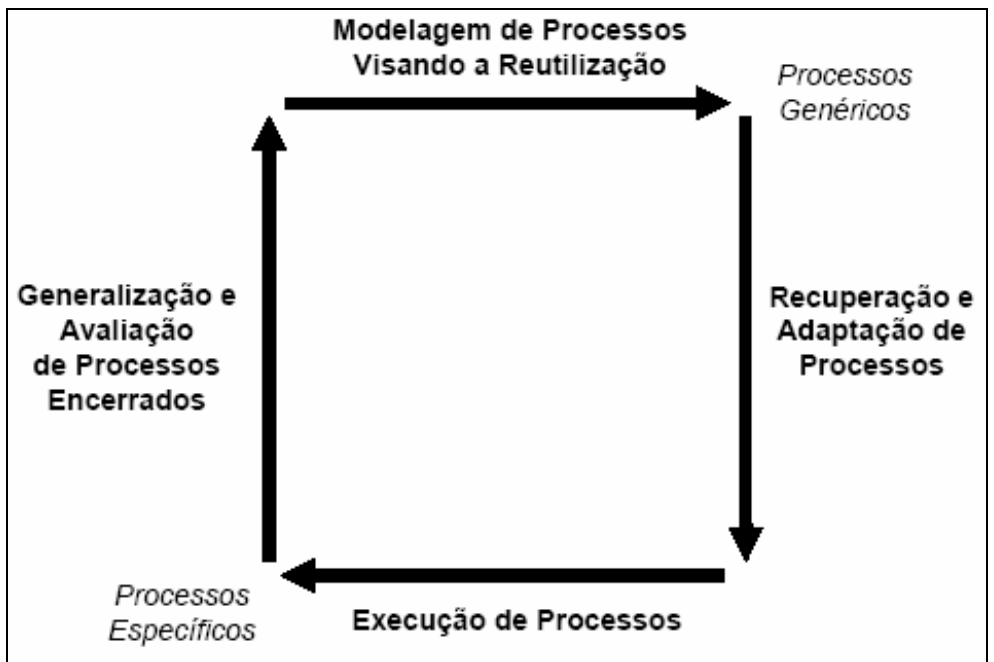

Figura 1 - Cenário Geral para Reuso de Processos de Software (adaptado de [Jørgensen 2001])

\subsection{Ambiente WebAPSEE: Histórico e Caracterização}

Desde 2005, o ambiente WebAPSEE [Costa 2006], originado a partir de uma experiência no desenvolvimento de um PSEE (Process-centerd Software Engineering Environment) chamado APSEE [Lima Reis 2003], vem sendo desenvolvido pelo Laboratório de Engenharia de Software (LABES) da Universidade Federal do Pará (UFPA). Tanto o software em si quanto a sua documentação estão disponíveis em LABES (2007).

O WebAPSEE fornece apoio automatizado para a gestão de processos de software, sendo projetado para permitir a integração de vários serviços relacionados com uma visão bastante ampla do meta-processo de software. Isto é, a ferramenta hoje apóia desde a concepção e levantamento de requisitos de processo até a geração de análise post-mortem dos processos, passando pelo controle da execução dos processos de forma bastante flexibilizada - implementando um modelo de execução descrito por Lima Reis (2003).

Vale ressaltar que o WebAPSEE já disponibilizava desde 2006 um mecanismo para prover a reutilização de processos através de uma função de cópia de processos, que reinicializa processos já existentes e atribui um novo identificador às cópias geradas. A Figura 2 mostra um exemplo em que um processo em execução (acima) cujas atividades (elipses) são decoradas com os estados da execução - é copiado, sendo gerado pelo sistema um novo processo "vazio" (abaixo), isto é, sem os detalhes da execução anterior. Porém, essa solução apresenta diversas limitações quanto aos requisitos para reutilização de processos de software, como: a inexistência de um registro sobre o histórico de reutilização, de mecanismos de adaptação e de análise sobre um processo e de uma representação explicitamente abstrata de um processo de software (templates) que permita a definição de processos padrões dentro da organização, seu histórico de modificações (versões) e instanciação desses como processos executáveis, entre outros. 


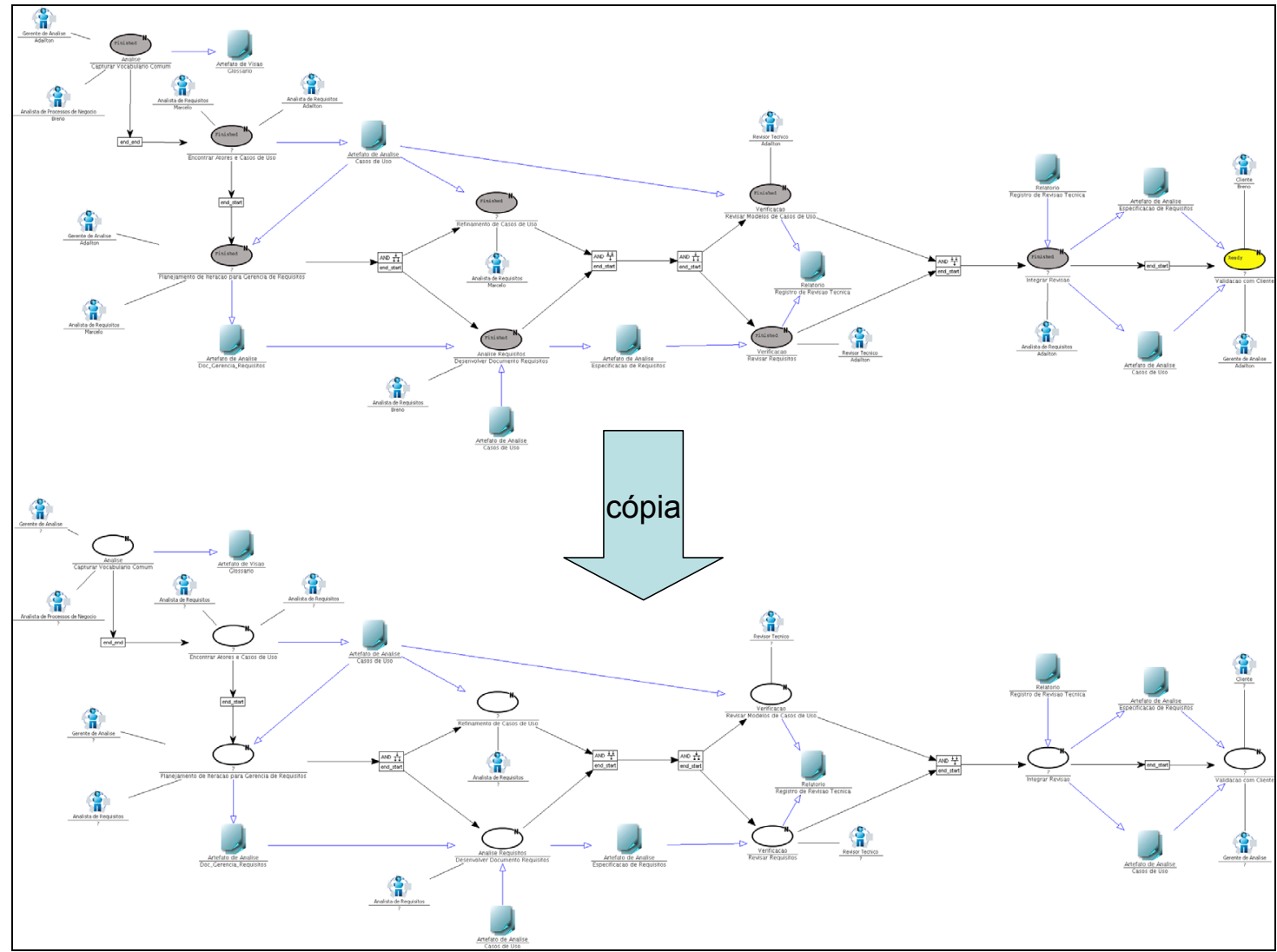

Figura 2 - Geração da cópia de um processo em execução

Nesse contexto, a ferramenta WebAPSEE-Reuse foi desenvolvida, a partir da segunda metade de 2006, com o objetivo de implementar a reutilização de processos dentro do ambiente WebAPSEE. Na realidade, o modelo e ferramenta apresentados aqui constituem uma segunda geração de um mecanismo para reutilização de processos que originalmente foi definido por Reis (2002).

\subsection{WebAPSEE-Reuse: Visão Geral das Funcionalidades Oferecidas}

A ferramenta WebAPSEE-Reuse é constituída por um conjunto de mecanismos computacionais para permitir a reutilização de processos de software. Para isso, foi adotada uma estrutura para representar modelos de processos abstratos (denominados templates), descrevendo seus dados, comportamentos e funcionalidades dentro do ciclo de vida do processo de software. Dessa forma, são propostos mecanismos de abstração para a modelagem de templates, uma máquina de estados para os templates e um conjunto de funcionalidades agregadas a esse modelo.

Um template deve ser compreendido como uma solução para um conjunto de problemas semelhantes, os quais não possuem informações relacionadas a uma organização específica. Um template pode ser customizado para atender os requisitos de um determinado contexto (metodológico, organizacional ou tecnológico), ou ainda combinado com outros templates ou modelos instanciados [Franch e Ribó 2002].

A figura 3 ilustra as principais funcionalidades do modelo proposto. A figura define dois conjuntos principais: na parte superior estão os processos (entidades concretas executáveis no ambiente e cuja realização resulta em software), enquanto que 
a parte inferior da figura ilustra os templates para reutilização (os quais constituem entidades abstratas - tais como os frameworks do Paradigma de Objetos). As funcionalidades principais são ilustradas pelas setas verticais da figura, as quais são usadas para nortear as situações possíveis no modelo. Tais funcionalidades são descritas a seguir:

- Process Instantiation ${ }^{1}$ é uma forma de reutilizar um modelo abstrato definindo processos (executáveis) como suas instâncias as quais podem ser adaptadas a um contexto específico;

- Process Composition é uma segunda forma de se reutilizar um modelo abstrato. Neste caso, o template é usado para compor um fragmento de um modelo de processo executável que está sendo definido;

- Process Distilling consiste na generalização de um modelo de processo encerrado, gerando um template correspondente.

Além das funcionalidades principais enumeradas acima, a ferramenta WebAPSEE-Reuse fornece como funcionalidades adicionais o Controle de Versões e a Padronização de Templates. O Controle de Versões possibilita a criação de versões de um template, permitindo o registro do histórico de modificações de um processo. Este recurso permite ainda que um template seja continuamente aperfeiçoado e, quando necessária, haja a substituição da versão atual por uma mais nova, mantendo as versões defasadas em um repositório. A Padronização de templates, por sua vez, é uma funcionalidade útil para promover um template em um modelo abstrato reutilizável (ou processo padrão), apoiando a definição de processos de acordo com os modelos de qualidade de processo de software.

A ferramenta WebAPSEE-Reuse também define mecanismos para recuperação, seleção e adaptação de processos. A recuperação e seleção constituem-se na adaptação da máquina de busca de processos do WebAPSEE, denominada WebAPSEESearchEngine [Sales 2006], para trabalhar no contexto dos templates e prover uma funcionalidade de visualização das informações de busca nos templates recuperados.

\footnotetext{
1 Em função do caráter internacional do projeto de desenvolvimento da ferramenta WebAPSEE é utilizada denominação em inglês para todas as suas funcionalidades.
} 


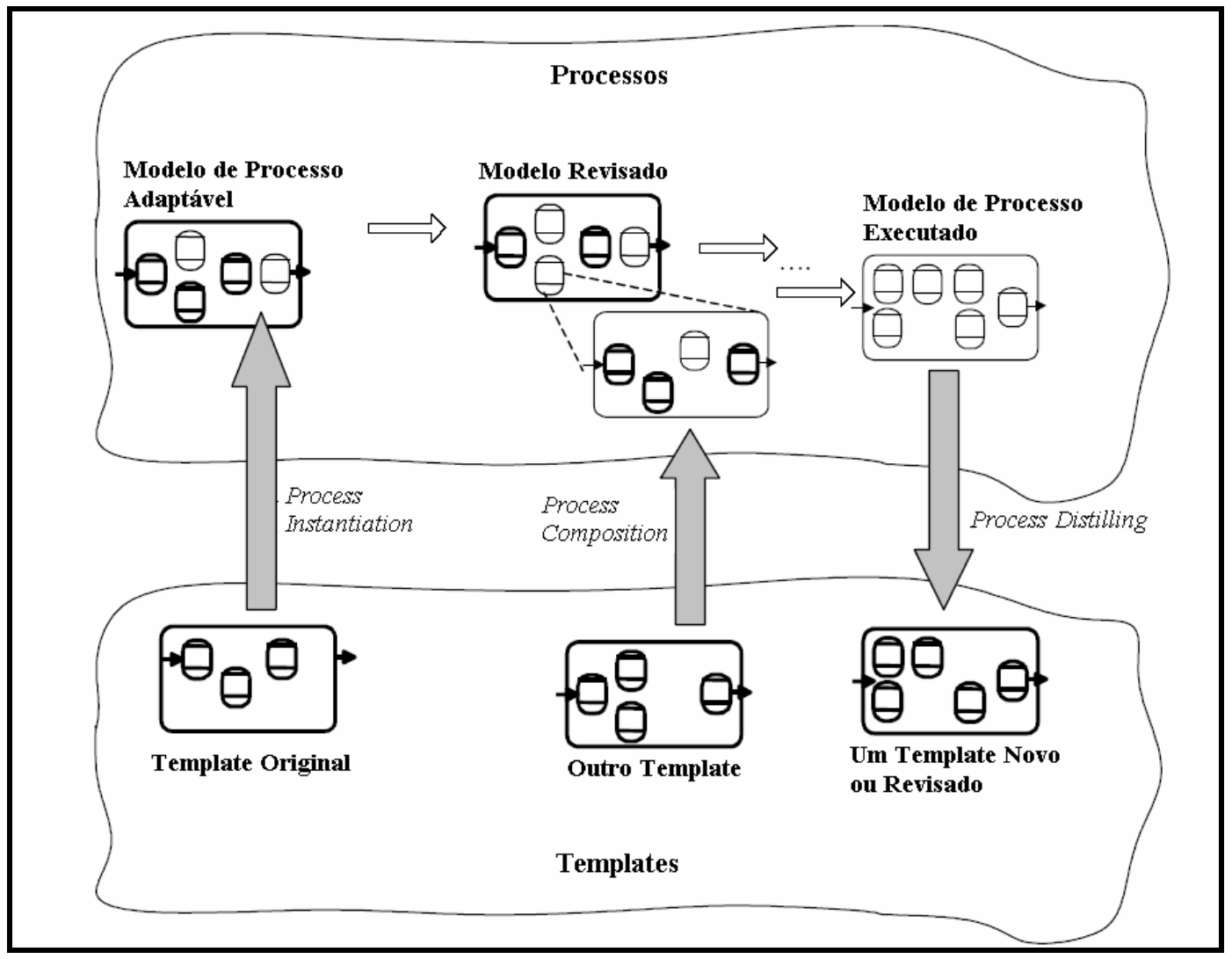

Figura 3 - Principais Funcionalidades do Modelo Proposto

Do ponto de vista do usuário esperado por este tipo de ferramenta normalmente associado às denominações de gerente ou engenheiro de processos em uma organização de desenvolvimento de software - a ferramenta WebAPSEE-Reuse constitui-se, de modo geral, da inclusão de novas funcionalidades ao ambiente existente. Essas funcionalidades estão concentradas em um menu denominado Process Reuse (Figura 4), salvo a funcionalidade de Composição de Processos, que possui outra forma para ser acessada, através do popup de uma atividade decomposta (isto é, uma atividade que pode ser definida por um subprocesso) no editor de modelagem de processos do ambiente WebAPSEE (Figura 5). Ao se selecionar a opção Process Composition, são disponibilizados para o usuário as funcionalidades para recuperação e adaptação de templates.

\begin{tabular}{|c|l|}
\hline & \\
\hline Process Reuse Tools Help & \\
\hline Templates & Create Template \\
\hline Process Instantiation & Open Template \\
Process Distilling & Save Template \\
Run Search Engine & To Become Defined \\
& Create New Version \\
\hline
\end{tabular}

Figura 4 - Menu Process Reuse 


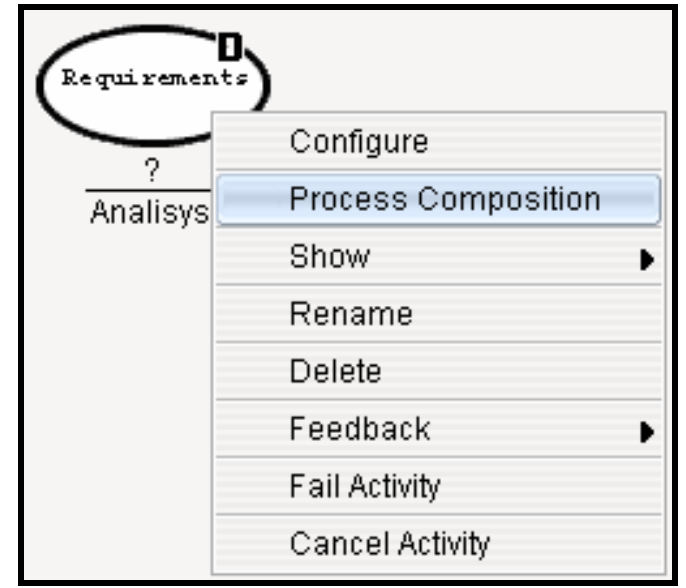

Figura 5 - Menu Popup de uma atividade decomposta

\subsection{Detalhamento Interno do Projeto e Implementação}

Para prover todas as funcionalidades citadas anteriormente foi criada uma máquina de estados para um template, a qual define as situações que podem ocorrer e ações possíveis a cada instante. Os estados de um template são:

- Draft (Rascunho), quando um template é criado, sendo possível modelá-lo ou modificá-lo;

- Defined (Definido), quando um template é padronizado em uma organização de desenvolvimento de software, impedindo sua alteração e permitindo sua reutilização;

- Pending (Pendente), quando é gerada uma nova versão de um template, indicando que a nova versão está sendo modelada e que a antiga versão não pode ser mais versionada;

- OutDated (Desatualizado), quando uma nova versão de um template é padronizada (no estado Defined), indicando que não pode ser mais reutilizado.

O mecanismo de versões proposto pela ferramenta é integrado ao modelo de estados apresentado acima, e é descrito a seguir. A partir do momento em que o usuário decide criar uma nova versão de um template, esse passa do estado Defined para o estado Pending e com isso é automaticamente criada uma nova versão (cópia) do template no estado Draft, permitindo que esse seja alterado. Porém, a nova versão ainda não é a versão corrente e ainda não poderá ser reutilizada - já que a mesma ainda não se constitui em um padrão (i.e., não foi promovida a tal pelo usuário). A partir do momento em que o usuário decidir tornar a nova versão um padrão da organização, a nova versão será promovida do estado Draft para o estado Defined e, em consequiência, a versão anterior será passada do estado Pending para o estado OutDated. Os templates no estado OutDated são mantidos no repositório para recuperação do histórico da organização e seus processos.

Do ponto de vista arquitetural, as regras de modelagem definidas para garantir que um template seja realmente um processo abstrato foram implementadas utilizando AspectJ [Kiczales 2001], uma extensão da linguagem Java para possibilitar a Programação Orientada a Aspectos. Essa escolha sobre a orientação a aspectos deveu- 
se, sobretudo, ao fato do editor gráfico do ambiente WebAPSEE ter sido originalmente construído para permitir somente a modelagem de processos concretos (executáveis). Dessa forma, facilita-se a modularização e a sincronização do código implementado com o editor de modelagem do WebAPSEE. Mais informações sobre o projeto e implementação deste mecanismo estão disponíveis em Costa e Sales (2007).

\section{Estudo de Caso}

Essa seção descreve o uso da ferramenta proposta em situações que envolvem a definição, reutilização e generalização de processos para problemas reais. Esse estudo foi desenvolvido com o objetivo de avaliar o modelo de reutilização proposto pela ferramenta WebAPSEE-Reuse. O referido estudo foi dividido em dois momentos, a definição do Rational Unified Process (RUP) como um template e a reutilização do mesmo em um contexto específico de um projeto de desenvolvimento de software.

O RUP define quatro fases genéricas, que devem ser adotadas para o desenvolvimento de software (Inception, Elaboration, Construction e Transition). Essas fases possuem iterações, em que devem ser produzidos resultados levando em consideração determinados níveis de detalhes. Além disso, inúmeras práticas (ou disciplinas) de Engenharia de Software devem ser seguidas em todas as fases do processo, sendo que o grau de comprometimento da prática com cada fase é representado através de oscilações.

Para a definição do template do RUP foi utilizada a linguagem de modelagem gráfica do WebAPSEE [Costa 2006] e as regras de modelagem definidas pelo modelo WebAPSEE-Reuse, representando-o como um modelo abstrato passível de ser reutilizado na definição de novos processos. Em síntese, o mapeamento do RUP para a linguagem de templates aqui proposta foi bem sucedida. Porém, algumas lições foram aprendidas, as quais são enumeradas a seguir:

- Dificuldade em se determinar o fluxo de controle para alguns workflows específicos, pois no RUP esses são orientados a artefatos e nem sempre são guiados pela sequiência das atividades;

- A versão atualmente implementada pela linguagem de modelagem de processos do WebAPSEE não possui representação para o conceito de ferramentas, usado com frequiência pelo RUP na sua descrição original;

- No RUP os artefatos possuem estados. Por exemplo, o artefato Use Case, que possui os estados outlined e described. No WebAPSEE isso não é aplicável visto que o ambiente é responsável pela execução e controle da manipulação e atualização dos artefatos. Portanto, não houve necessidade de representação de estados para representar as modificações de artefatos no mapeamento do RUP para templates.

Após essa modelagem, esse template foi reutilizado para guiar o processo de desenvolvimento de software aplicado na disciplina de Laboratório de Engenharia de Software no primeiro semestre de 2007 dos cursos de Bacharelado em Ciência da Computação e Bacharelado em Sistemas de Informação da Universidade Federal do Pará.

Primeiramente, os modelos foram instanciados a partir do template do RUP original, conforme as lições apresentadas acima. Em seguida, os modelos foram 
adaptados devido ao caráter simplificado da disciplina. Por exemplo, da fase de Teste do modelo RUP original foi excluída a atividade de identificação de motivações de testes, pois a mesma poderia se tornar um entrave burocrático ao andamento do projeto. A figura 6 apresenta o fragmento do processo relativo à fase de Teste. Decisões similares foram tomadas com outras atividades e artefatos do modelo, além da junção de todos os diagramas definidos em UML em um único artefato, o que é feito de forma separada no RUP.

Por fim, após a execução, esse modelo foi generalizado, gerando um novo template que inclui todas essas alterações, que pode ser útil para servir de base nas próximas turmas da disciplina mencionada no futuro. Vale ressaltar também que o modelo proposto pela ferramenta possibilitou uma interpretação diferenciada das possibilidades definidas pelo RUP. Portanto, além das variações originalmente previstas pelo RUP, denominadas como "Completo" e "Simplificado", outras variações são proporcionadas pela ferramenta. Dessa forma, a ferramenta ofereceu flexibilidades em relação à reutilização do processo aplicado na disciplina citada anteriormente, pois com a mesma foi possível:

- Excluir atividades, artefatos, cargos e recursos indesejados do modelo de processo reutilizável;

- Incluir novas atividades relevantes e mais apropriadas a um contexto específico;

- Modificar atividades, artefatos, cargos e recursos tornando-os mais adequados ao contexto em questão;

- Reunir um conjunto de artefatos em um único artefato, assim como cargos e atividades.

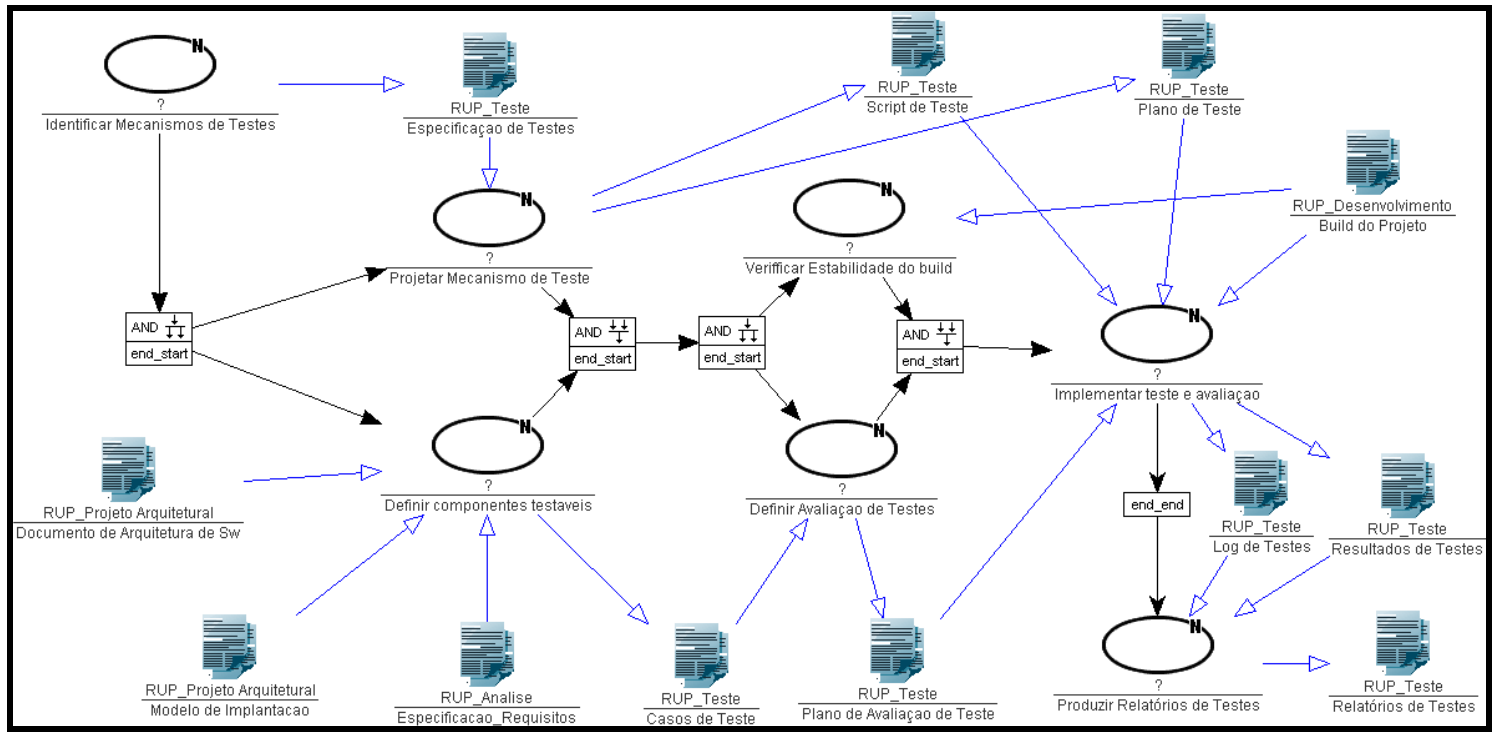

Figura 6 - Fase de Teste adaptada do modelo RUP

\section{Trabalhos Correlatos}

Nesta seção são apresentados dois trabalhos relacionados ao exposto nesse artigo e que se destacam atualmente na área de Reutilização de Processos de Software. Embora existam outros trabalhos similares, citados por [Reis 2002] e [Costa e Sales 2007] 


\subsection{Rational Method Composer}

O Rational Method Composer (RMC) [RMC 2007] é um produto comercial da IBM construído sob a plataforma de desenvolvimento Eclipse [ECLIPSE 2007] que evoluiu de uma linha de produtos anterior composta pelas ferramentas IBM Rational Process Workbench, do RUP Organizer e do RUP Builder. RMC é uma plataforma de ferramentas que permite que engenheiros e gerentes de processo implementem, desenvolvam e façam a manutenção de processos para organizações ou projetos individuais.

A proposta do RMC é ajudar nos dois problemas principais para a implantação com êxito de novos processos: instruir as equipes de desenvolvimento sobre os métodos aplicáveis às funções pelas quais são responsáveis e fazê-los compreender como aplicar esses métodos durante todo o ciclo de vida do desenvolvimento. Métodos aqui podem ser entendidos como templates sob a ótica do WebAPSEE-Reuse.

A reutilização de componentes é permitida através dos seguintes recursos:

- Uso do mecanismo de plug-in para possibilitar os recursos de reutilização e capacidade de extensão dos componentes do método. Plug-ins são agrupamentos de componentes de processos de software;

- Apoio para definição de padrões de processo reutilizáveis e vinculados a partir de boas práticas para rápida montagem do processo através de simples operações de arrastar e soltar.

Dessa forma, o RMC é uma ferramenta comercial de engenharia do processo de software de propósito geral com recursos para autoria, configuração, visualização, e publicação na Web/Intranet de métodos e processos. Além disso, a ferramenta provê a reutilização de componentes usando o conceito de plug-ins. Por outro lado, o RMC não fornece uma visualização geral dos componentes de processos (somente de categorias de componentes isoladamente), e apresenta uma complexa estrutura de recursos associada que dificulta seu entendimento. Não há um editor gráfico de processos - a modelagem é realizada essencialmente através do preenchimento de tabelas onde, embora uma representação gráfica não editável do processo possa ser gerada automaticamente pela ferramenta. O RMC não executa processos, o que impede a sua adoção para automatizar uma porção mais significativa do meta-processo de software.

Por sua vez, o WebAPSEE-Reuse é uma ferramenta livre fortemente baseada em uma notação gráfica para edição de processos. A solução aqui proposta encontra-se integrada a um ambiente de gestão de processos - o WebAPSEE - e isto tem como consequiência positiva a obtenção de todos os benefícios associados com as demais etapas do ciclo de vida de processos (i.e., modelagem, simulação, instanciação, avaliação, execução, entre outras etapas). Por outro lado, não se deve deixar de mencionar a complexidade inerente associada à implantação de um PSEE em uma organização de desenvolvimento de software, ao contrário da abordagem menos ambiciosa oferecida pelo RMC.

Por fim, o RMC se destaca pelas facilidades oferecidas na geração de webguides, isto é, guias eletrônicos baseados nos padrões da WWW para documentar os modelos de processo de software gerados. Em contrapartida, o WebAPSEE possui o seu foco na geração de relatórios gerenciais a partir da execução de processos, não fornecendo grandes facilidades para disseminação dos processos em si. 


\subsection{Estação TABA: Def-Pro e Adapt-Pro}

A Estação TABA é um PSEE capaz de gerar, através de instanciação, ambientes de desenvolvimento de software (ADS) adequados às particularidades de processos de desenvolvimento e projetos específicos [Rocha 1990]. O referido ambiente é resultado do projeto TABA desenvolvido pela COPPE-Sistemas da Universidade Federal do Rio de Janeiro.

Segundo a documentação disponível em [TABA 2007] duas ferramentas integradas a Estação TABA apóiam a reutilização de processos de software: DEF-PRO, que apóia a definição de processos padrão e de processos instanciados; e Adapt-Pro, que apóia o gerente de projetos durante a adaptação do processo utilizando técnicas de gerência do conhecimento.

Def-Pro [Rocha 2000] trata-se de uma ferramenta de apoio à definição de processos de software padrão para uma organização, com base na norma ISO/IEC 12207, nos modelos de maturidade (como CMM e a ISO/IEC 15504), nas características da organização e no tipo de ADS para o qual está sendo definido o processo. Uma das características dessa ferramenta é que o usuário é obrigado a seguir um dos padrões previamente definidos (ISO/IEC 12207, 15504, CMM, MPS.BR) ou combinações dos mesmos. Dessa forma, Def-Pro não fornece recursos para autoria de processos-padrão ad-hoc (não seguindo processos ou metodologias pré-existentes) nem seguindo outros modelos de desenvolvimento como o RUP (ou similares). Além disso, apesar da ferramenta permitir a modificação de processos-padrão por meio de combinações das normas e modelos, tais modificações não são registradas.

Já o WebAPSEE-Reuse fornece recursos para autoria livre, possibilitando a definição de tais modelos e normas de forma manual, os quais podem ser adaptados a contextos específicos. Um mecanismo de gestão de configuração de processos-padrão que guarda as modificações inseridas nesses processos também é fornecido pela ferramenta proposta.

Adapt-Pro [Berger 2003], por sua vez, trata-se de uma ferramenta que possibilita a utilização do conhecimento organizacional armazenado no repositório da organização quando um novo projeto é definido e torna-se necessário adaptar o processo especializado ao projeto. Na prática, essa ferramenta apóia as atividades de caracterização do projeto, planejamento do processo e instanciação de um ADSOrg, definidas no processo de instanciação proposto pela Estação TABA. Essa ferramenta pouco explora a recuperação e análise post mortem de projetos previamente executados, o que se torna importante para a utilização das experiências obtidas em projetos passados proposto pela ferramenta. Em oposição, a abordagem adotada pelo WebAPSEE-Reuse define mecanismos de recuperação (com a definição de uma máquina de busca de templates), e generalização (em que processos executados com sucesso podem ser “destilados” para a geração de novos templates Reutilizáveis).

\section{Considerações Finais}

Esse artigo descreve a ferramenta WebAPSEE-Reuse como um apoio sistemático na reutilização de processos de software, buscando prover a melhoria contínua de processos, visto que as informações tecnológicas e gerenciais de projetos passados servem de base para desenvolvimento de novos projetos. Deve-se observar que [Costa e 
Sales 2007] apresenta um detalhamento maior acerca do mecanismo aqui proposto, ilustrando o mapeamento mais completo dos modelos de processos RUP e Programação Extrema [Beck 2005] para a notação proposta.

A ferramenta WebAPSEE-Reuse atende a algumas exigências de modelos de qualidade de software como o CMMI, ISO-SPICE e o MPS.BR, quanto à gestão de configuração de processos, definição de processos padrões, reutilização de processos, entre outros.

Quanto ao MPS.BR, a ferramenta WebAPSEE-Reuse atende, mais especificamente, a dois processos, Adaptação do Processo para Gerência do Projeto e Definição do Processo Organizacional. O primeiro processo é atendido pela ferramenta através da customização e composição de processos, presentes nas funcionalidades Process Instantiation e Process Composition, enquanto que o segundo processo é atendido através do estabelecimento e da manutenção de um conjunto de processos padrões da organização, disponíveis através das funcionalidades de Padronização de Templates e Controle de Versões.

O WebAPSEE-Reuse trata-se de uma solução diferenciada, pois se constitui em uma solução livre, está em um ambiente integrado (WebAPSEE) que provê soluções como, gestão de processos de software executáveis, gestão de configuração de artefatos, entre outros, não está preso a um conjunto específico de normas, permitindo flexibilidade, além de possibilitar a definição de processos com maior nível de detalhes.

Convém ressaltar que o modelo de reutilização de processos adotado pelo WebAPSEE é ainda composto por um elemento adicional denominado Políticas de Processo. As Políticas são elementos ortogonais aos processos e templates que servem para expressar propriedades sintáticas ou estabelecer regras para alocação de pessoas e recursos na execução de processos [Reis 2002]. Do ponto de vista de apoio à reutilização de processos, as Políticas são úteis para estabelecer características de um template original que devem ser obrigatoriamente mantidas nas suas instanciações. Maiores informações sobre o modelo de Políticas atualmente adotado pelo WebAPSEE podem ser encontradas em [Silva 2007].

$\mathrm{O}$ trabalho atual prossegue com o uso e aperfeiçoamento da infra-estrutura desenvolvida para utilizá-la como instrumento para a descrição e experimentação de um modelo de processo de software para ser implantado pelo Centro de Tecnologia da Eletronorte, sediado em Belém-PA. Houve treinamento dos profissionais da empresa com o uso da ferramenta e, atualmente, no momento de submissão deste artigo, um projeto-piloto vem sendo executado com o desenvolvimento de um sistema de software tendo por base uma primeira versão do modelo de processo.

\section{Agradecimentos}

Este trabalho foi apoiado pelo CNPq (processo 550451/2003-0) e ELETRONORTE (projeto número 50528 do ciclo de P\&D 2003/2004).

\section{Referências}

Beck, Kent. (2005). "Programação Extrema Explicada: acolha as mudanças". Porto Alegre: Bookman. 
Berger, P. M. (2003). "Instanciação de Processos de Software em Ambientes Configurados na Estação TABA". Tese de Mestrado. Rio de Janeiro: PPGE da COPPE/UFRJ.

Costa, Anderson J. S. et al.(2006). "Gerência Flexível de Processos de Software com o Ambiente WebAPSEE", In Simpósio Brasileiro de Engenharia de Software, 20., Florianópolis: Informática-UFSC, v.1.

Costa, Anderson; Sales, Ernani (2007). "Uma proposta para Reutilização de Processos de Software para o ambiente WebAPSEE". Trabalho de Conclusão de Curso. Belém: Bacharelado em Ciência da Computação - UFPA. Disponível em http://www.labes.ufpa.br/.

Dorling, A., El Emam, K, Drouin, J., Melo, W. (1997) SPICE: The Theory and Practice of Software Process Improvement and Capability Determination. Wiley-IEEE Computer Society Press.

ECLIPSE (2007). “Eclipse - an open development platform”, http://www.eclipse.org, Junho.

Fiorini, S. T. (2001) “Arquitetura para Reutilização de Processos de Software”, $243 \mathrm{f}$. Tese de Doutorado. Rio de Janeiro: Departamento de Informática da Pontifícia Universidade Católica (PUC-RJ).

Franch, X.; Ribó, J. (2002) "Supporting Process Reuse in PROMENADE". Research Report, No. LSI-02-14-R. Barcelona: Departament de Llenguatges i Sistemes Informàtics, Universitat Politécnica de Catalunya.

Fuggetta, Alonso (2000). "Software Process: A Roadmap". In Finkelstein (Ed.), Future of Software Engineering. New York: ACM Press.

Jørgensen, H.; Carlsen, S. (2001). "Writings in Process Knowledge Management: Management of Knowledge Captured by Process Models". Technical Report, No. STF40 A00011. Oslo: SINTEF Telecom and Informatics.

Kiczales, Gregor et al. (2001). "Aspect-Oriented Programming”. In Proceedings of the European Conference on Object-Oriented Programming (ECOOP). Finland. Springer-Verlag LNCS 1241.

Krueger, C. W. (1992). "Software reuse". In ACM Computing Surveys 24, 2 (Jun. 1992), 131-183.

Kruchten, P. (2000). “The Rational Unified Process: An Introduction”. 2nd ed. [S.1.]: Addison-Wesley.

Labes (2007). "Laboratório de Engenharia de Software da Universidade Federal do Pará", http://www.labes.ufpa.br

Lima Reis, Carla A. (2003) "Uma Abordagem Flexível para Execução de Processos de Software Evolutivos". Tese de Doutorado. Porto Alegre: PPGC da UFRGS.

Mohagheghi, P., et al. (2004). "An Empirical Study of Software Reuse vs. DefectDensity and Stability". In Proceedings of the 26th international Conference on Software Engineering (May 23 - 28, 2004). International Conference on Software Engineering. IEEE Computer Society, Washington, DC, 282-292. 
Pressman, Roger S. (2005) "Software Engineering: a practioner's approach". McGrawHill, $6^{\text {th }}$ edition.

Rational (2007) “IBM Rational Software”, http://www.rational.com, Março.

Reis, Rodrigo Q. (2000). "Reutilização de Processos de Software”. Exame de Qualificação EQ-46. Porto Alegre: PPGC da UFRGS.

Reis, Rodrigo Q. (2002). “APSEE-Reuse: Um Meta-modelo para Apoiar a Reutilização de Processos de Software”. Tese de Doutorado. Porto Alegre: PPGC da UFRGS.

RMC (2007). "Rational Method Composer", http://www306.ibm.com/software/awdtools/rmc/, Janeiro.

Rocha, A. R. C. et al (1990). "Taba: A Heuristic Workstation for Software development", In Proceedings of COMPEURO 90, Tel Aviv, Israel, Maio.

Rocha (2000). "Def-Pro: Uma ferramenta para Apoiar a Definição de Processos Padrões". In Simpósio Brasileiro de Qualidade de Software. João Pessoa, Brasil.

RUP (2007). "IBM Rational Unified Process", http://www304.ibm.com/jct03004c/businesscenter/smb/us/en/solutionsummary/xmlid/29811/na v_id/product, Janeiro.

Sales, Ernani O. et al. (2006) "Uma Ferramenta para Recuperação de Modelos de Processo de Software Reutilizáveis". In Simpósio Brasileiro de Engenharia de Software, 20., Florianópolis: Informática-UFSC.

SEI (2007). "Welcome to the CMMI Web Site", http://www.sei.cmu.edu/cmmi/, Janeiro.

Silva, M.A. (2007). "WebAPSEE-Planner - auxílio à alocação de pessoas em projetos de software através de políticas". Trabalho de Conclusão de Curso. Belém: Bacharelado em Ciência da Computação - UFPA. Disponível em http://www.labes.ufpa.br, Junho.

SOFTEX (2007). "Mps.Br - Melhoria de Processos do Software Brasileiro", http://www.softex.br/mpsbr/, Janeiro.

TABA (2007). "ESTAÇÃO TABA: Ambiente de Desenvolvimento de Software", http://ramses.cos.ufrj.br/taba/, Junho. 